\title{
Analysis of Principal Stress Projection in Femur with Total Hip Ar- throplasty using CT-image Based Finite Element Method
}

\author{
Kosei Fukuoka ${ }^{1}$ and Mitsugu Todo ${ }^{2 *}$ \\ ${ }^{1}$ Interdisciplinary Graduate School of Engineering Sciences, Kyushu University, Japan \\ ${ }^{2}$ Research Institute for Applied Mechanics, Kyushu University, Japan
}

*Corresponding author: Mitsugu Todo, Associate Professor, Research Institute for Applied Mechanics, Kyushu University, 6-1 Kasuga-koen, Kasuga, Fukuoka 816-8580, Japan, Tel: +81-92-583-7762, Fax: +81-92-583-7762

\begin{abstract}
The research demonstrates a novel three-dimensional finite element analysis to analyze biomechanical changes in a femur with implant. The method uses principal stress projections to determine trabeculae trajectories in the femur. Visual comparisons of the projections of the femurs show areas of cortical and cancellous changes due to changes in tensile and compressive stresses, revealing areas of stress shielding. The method also includes a bone remodeling algorithm to simulate bone adaptation. In the experiment, a patient-specific femur model with inhomogeneous material properties was created from CT scans. Models of femurs with implants varying in type and size were also created. Maximum load during walking was simulated with realistic muscle forces, and stress projections of the femur model were compared to trabecular trajectories of a real femur for validation. Bone remodeling was also simulated to investigate changes in projections over time. High correlation was found between the principal stress projections of the computational model and trabeculae trajectories of a real femur. Changes in projections were evident for implant models, suggesting stress shielding and bone remodeling. The analysis provides an effective method for planning implants that are ideal for patients and for designing future implants that preserves the biomechanics of the femur to maintain its physiology.
\end{abstract}

\section{Keywords}

Biomechanics, Finite element analysis, Total hip arthroplasty, Bone remodeling

\section{Introduction}

Total hip arthroplasty (THA) is a type of joint replacement surgery that has been the standard treatment for patients with osteoarthritis, avascular necrosis, and/or fracture within the hip joint. In THA, both the acetabu- lum and the femoral head are replaced, and three components are required: A stem and a spherical head that replaces the femoral head, and a hemispherical cup with an inner liner to replace the acetabulum. While fundamental concept of hip arthroplasty has changed little since its appearance in medicine, the implants have experienced numerous changes in terms of material and design [1]. During the early stages of development, the THA implant had a metal-on-metal (MOM) bearing combination. All components were made cobalt-chrome alloy and bone cement was used to fixate the implant to the bone. The implant head was also similar in size as the anatomical head, giving the hip a natural physiological range of motion (ROM). When it was revealed that MOM produced high frictional torque, metal-on-polymer became the popular bearing combination choice. It soon became evident that debris from the plastic liner due to volumetric wear led to osteolysis and causing the implant to loosen. Thus, the implant head size was reduced to decrease the wear, which also decreased the ROM of the hip, limiting the patient's mobility. The next fifty years saw the introduction of two more bearing combinations (ceramic-on-polymer and ceramic-on-ceramic), though much of the implant designs and the small head size remained unchanged. Presently, hip implants are available in all bearing combinations and in various shapes and sizes. THA implants with large head size are also becoming popular, as researches have shown that with larger head sizes, lubrication regime changes from boundary lubrication to fluid-film lubrication [2]. New researches in materials have also led to bearings having very low frictional coefficients. In addition, porous coat-

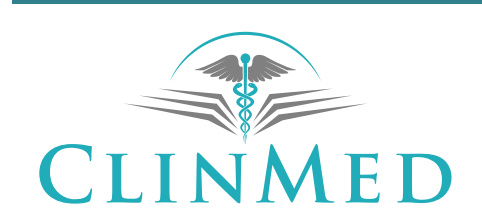

INTERNATIONAL LIBRARY
Citation: Fukuoka K, Todo M (2018) Analysis of Principal Stress Projection in Femur with Total Hip Arthroplasty using CT-image Based Finite Element Method. Int Arch Orthop Surg 1:003.

Accepted: September 18, 2018; Published: September 20, 2018

Copyright: (c) 2018 Fukuoka K, et al. This is an open-access article distributed under the terms of the Creative Commons Attribution License, which permits unrestricted use, distribution, and reproduction in any medium, provided the original author and source are credited. 
ings around the stem have led to the development of cement less implants, providing stronger bone fixation than using bone cement.

However, there are still risks to hip arthroplasty and thousands of revision surgery is conducted each year. The main indications for surgery that concerns the implant are aseptic loosening, dislocation, and the wear of acetabular component [3]. The main risk factors are can be categorized as being mechanical, biological, or biomechanical. Mechanical risk factors include incorrect determination of implant size, position, and orientation. As mentioned earlier, using a smaller implant head size decreases the ROM of the hip and increases the risk of the implant impinging with the cup, leading to dislocation [4,5]. Biological risk factors are derived from improper choice of implant type. With implants using bearing combinations of a polymer, the wear particles are associated with osteolysis, causing aseptic loosening of the implant. With MOM implants, the release of metal ions has been linked to metallosis. In addition, the size of head has been known to coincide with volumetric wear, with increasing head size leading to greater wear [6]. Both mechanical and biological risks have been heavily discussed in literature, and with proper clinical assessment and pre-surgical planning, the occurrence of these risks could be minimized. However, biomechanical risk factors are based on the remodeling of the bone, or the biological response due to mechanical change. Stress shielding is one factor in which the body load is shifted from the femur to the implant, causing osteopenia [7-11]. Such condition can cause aseptic loosening due to instability in fixation, or cause sudden fatal damage to the femur, such as fracture. In order to clinically assess biomechanical risks from an engineering perspective, researchers have focused on the bone adaptation theory [12-18].

The first bone adaptation theory, known as "Wolff's Law" suggests that the bone adapts to changes in stress and tries to minimize load. Wolff also illustrated his version of principal stress projections and postulated the "Trajectorial Theory", in which he noted that cancellous bone architecture is formed of a network of perpendicular intersections [19]. While Wolff's Law has remained as the philosophy of bone remodeling, the Trajectory Theory has been dismissed by modern research, as it has been found that the cancellous architecture is non-orthogonal, and its orientation is governed clearly by loading [20].

Finite element analysis (FEA) has frequently been used to simulate physiology of the femur, using patient-specific three-dimensional (3D) models made from computed tomography (CT) scan and inhomogeneous material properties calculated from the Hounsfield unit (HU) of the CT scan [21]. The use of FEA has allowed researchers to examine stresses and strains and to conduct risk assessments. Recently, the push for realism has introduced the use of telemetry devices to determine hip contact force in real time. Combining the data with musculoskeletal modeling has allowed researchers to mimic realistic physiological loading conditions [22-25]. Numerical and computational bone adaptation theory has also been suggested, following three basic concepts: Alteration in bone architecture due to mechanical stimulus, calculation of updated material property, and calculation of new stress and strain fields from updated material properties [26-30]. Computationally derived principal stress projections have also been evaluated with trabeculae structure of the femur with success [31,32].

Despite of the advancements in materials and designs, hip implants continue to cause complications. The National Joint Registy (NJR) for England and Wales, which has a large population and high rates of coverage, have recently revealed that the revision rate for MOM implants, specifically HRA implants, was much higher than other bearing combinations [33,34]. The findings have led to government health agencies in England and the United States to release alerts on the use of particular hip implants. Furthermore, within the past 10 years, major implant companies have recalled their implants. While researches concerning implant design have been conducted, most have been based on minimizing mechanical and biological risks. Patient-specific implants have also been simulated; however, the advantages have been minor compared to the cost and time to manufacture such implants [35-39]. Furthermore, long-term safety has not been addressed, which is a key factor for femur and implant survival, as well as the quality of life for the patient. Ideally, there should be minimal differences between the biomechanics of an intact femur and that of a femur with implant. Any alterations in loading will affect the stress and strain distribution, which will cause respective changes in the trabecular structure and henceforth the bone density. Thus, the primary design criteria of an implant must be based on minimizing biomechanical risks, followed by the minimization of mechanical and biological risks. In order to assess the effectiveness and safety of the implant design, it is necessary to first replicate the biomechanics of the femur, test under realistic physiological conditions, followed by bone remodeling simulation to predict long-term outcome. To the best of our knowledge, the combination of the three works has not yet been reported in literature.

The objectives of this study were to realistically simulate the physiological condition of the femur, to investigate the effects of an implant in the femur, and to utilize the obtained results for optimization of implant designs.

\section{Finite Element Modeling}

\section{D models}

CT scans $(512 \times 512,1 \mathrm{~mm}$ slices $)$ from a male 54 


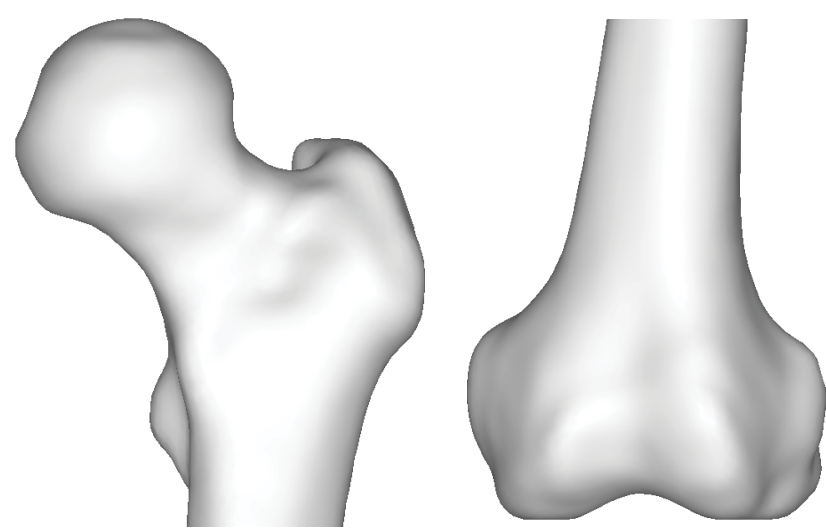

Figure 1: Intact bone model.
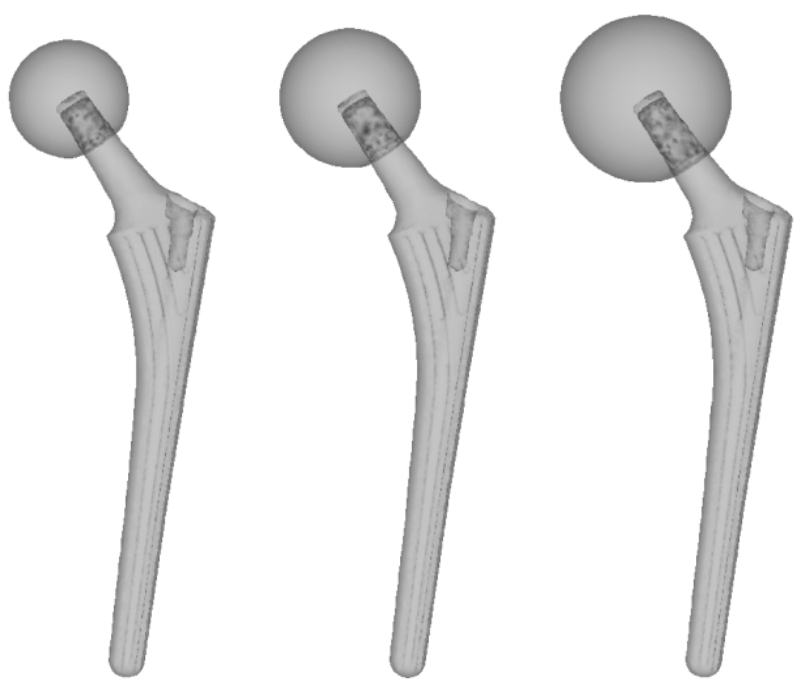

Figure 2: THA implant ( $\varnothing 34 \mathrm{~mm}, \varnothing 40 \mathrm{~mm}$, and $\varnothing 48 \mathrm{~mm})$.

yr patient were used to make an intact bone model as shown in Figure 1. MECHANICAL FINDER 6.1 (Research Center of Computational Mechanics Inc., Japan) (MF) was used to trace region of interest (ROI) around the outer cortical region on the scans to obtain the anatomical structure of the left femur. The ROls were then stacked and the gaps between the CT scans were automatically conjoined by the software to create a femur volume. The volume was surface meshed (unstructured linear triangular mesh) using ANSYS ICEM CFD v13 (ANSYS Inc., USA). Rapidform XOR3 (INUS Technology Inc., USA) was used to smooth the meshed model, and FEMAP 10.1.1 (Siemens PLM Software, USA) was used for all editing made on the meshed model. The final volume was remeshed (unstructured linear tetrahedral mesh) in MF.

CAD models of THA implant were directly imported into MF. The THA implant consisted of a $156 \mathrm{~mm}$ length stem and a $\varnothing 34 \mathrm{~mm}$ head. To assess variations in implant size, the THA implant was modified to include four additional models; two with increased head sizes; $\varnothing 40$ $\mathrm{mm}$ and $\varnothing 48 \mathrm{~mm}$ as shown in Figure 2; and two with decreased stem length; $75 \%$ and $50 \%$ as shown in Figure 3. Thus, a total of six implant models were created. The implants were positioned in a computational steer-
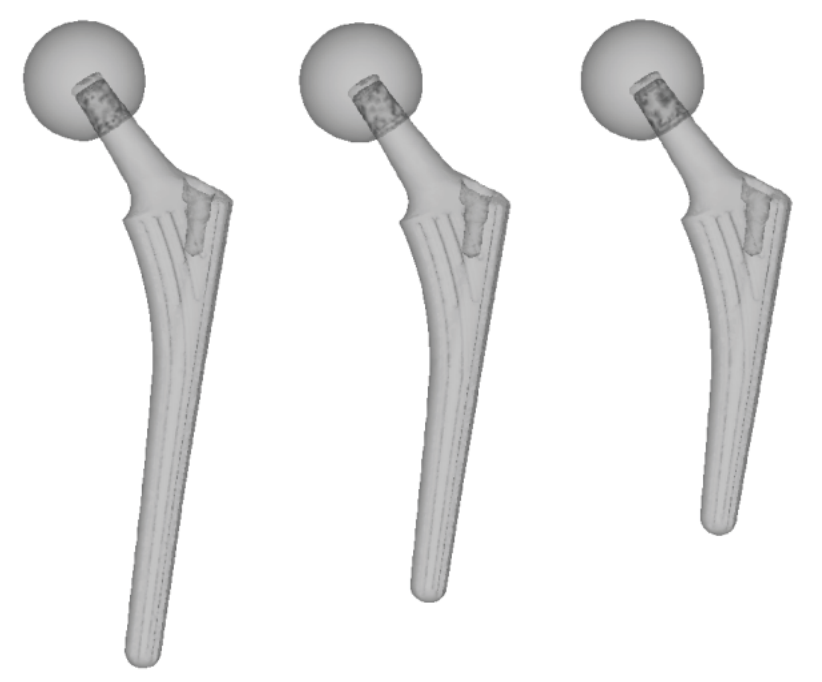

Figure 3: THA implant (100\%, $75 \%$, and $50 \%$ stem length).

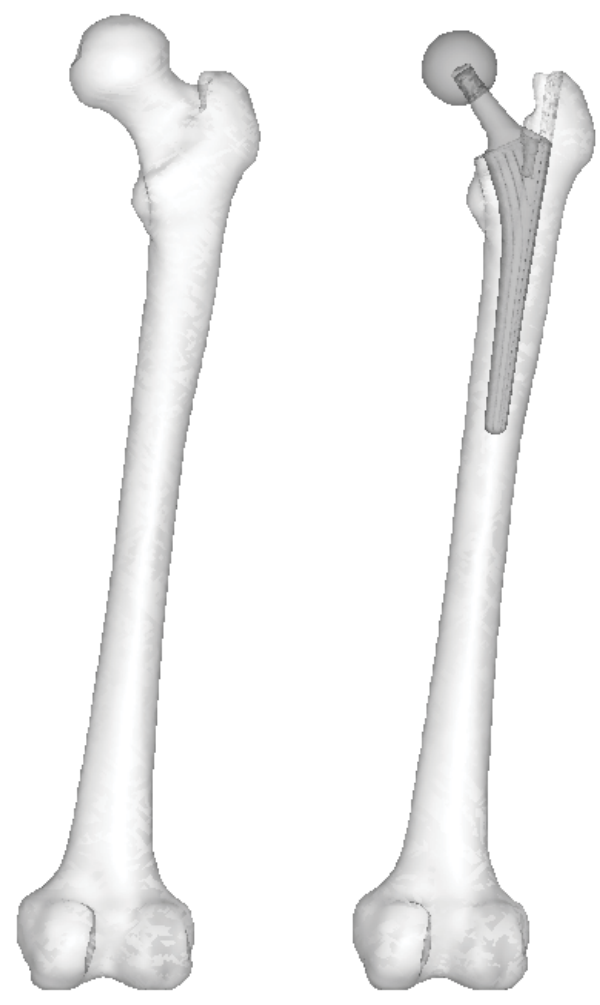

Figure 4: Intact bone and THA model.

ing environment as shown Figure 4. All models were meshed with linear unstructured tetrahedral mesh.

\section{Material properties}

Material properties of the femur were derived from the ash density extracted from the HU of the CT scans using the following formula:

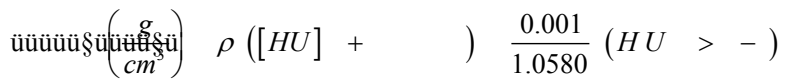

$$
\begin{aligned}
& \text { Density }\left(\frac{\mathrm{g}}{\mathrm{cm}^{3}}\right)=0(\text { H.U. } \leq-1)
\end{aligned}
$$

The Young's modulus and the yield stresses were calculated from the ash density as proposed by Keyak, et al. [21]. The Poisson's ratio was set to 0.3. For the THA implant, titanium alloy was assigned to the stem with 
Table 1: Physiological loading during walking.

\begin{tabular}{|l|l|}
\hline Type of load & Forces $(\mathbf{N})$ \\
\hline Intersegmental resultant & 483.19 \\
\hline Abductor & 632.30 \\
\hline Tensor fascia late, proximal & 115.20 \\
\hline Tensor fascia late, distal & 115.38 \\
\hline Vastus lateralis & 574.64 \\
\hline
\end{tabular}

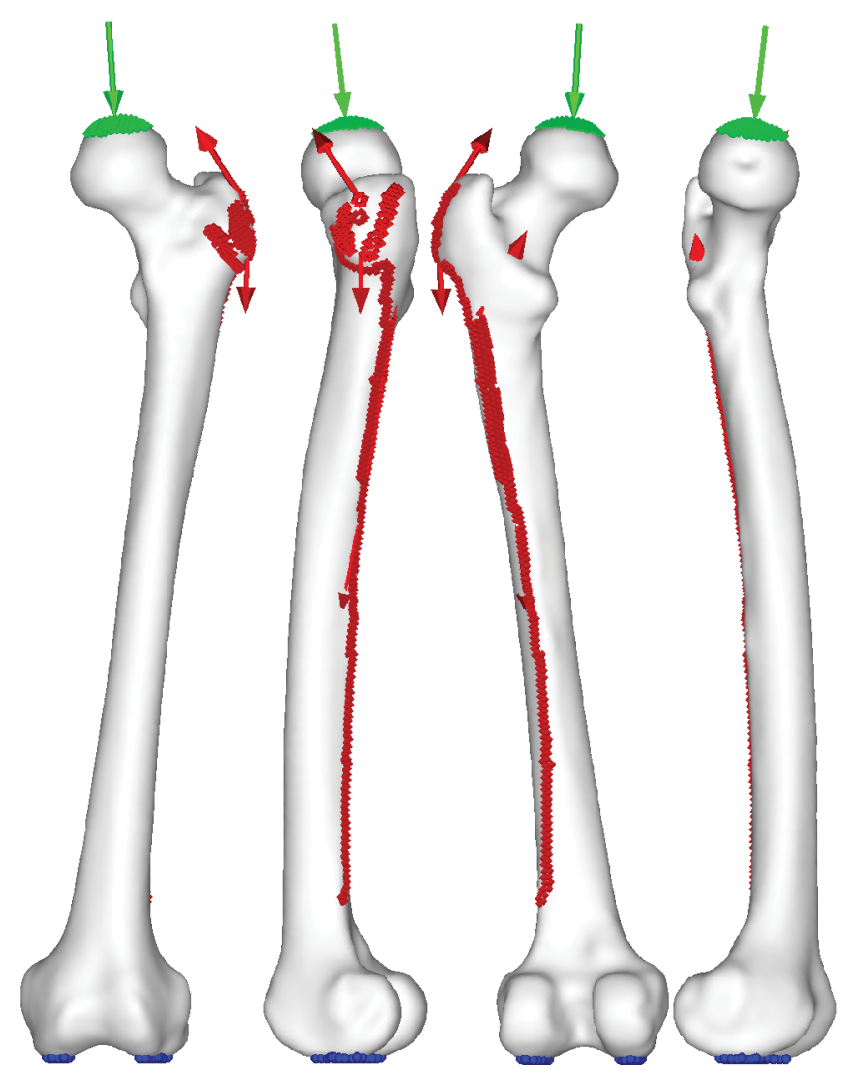

Figure 5: Walking contact force.

Intersegmental resultant $=$ green $\mid$ muscle $=$ red $\mid$ constraint $=$ blue.

the Young's modulus of $114 \mathrm{GPa}$ and the Poisson's ratio of 0.34 , and alumina to the head with the Young's modulus of $370 \mathrm{GPa}$ and the Poisson's ratio of 0.32 .

\section{Boundary conditions}

Simplified maximum walking (Table 1) contact forces determined by Heller, et al. were applied to the models [40]. Each \% body weight (BW) was configured with the patient's BW. Modifications were made on the locations of the origin and insertion of the muscle, as well as the distributions, for a realistic anatomical representation. Simplified abductor muscles were expanded to include gluteus medius, gluteus minimus, and gluteus maximum to the correct anatomical position and distribution as shown in Figure 5 . The porous coating on the upper half of the implant was assumed to be fully bonded with the bone as expressed in Figure 6. Non-porous areas were assumed to have a friction coefficient of 0.2 . Fixed constraint was applied to the distal femur.

\section{Bone adaptation algorithm}

Bone adaptation algorithm proposed by Huiskes, et

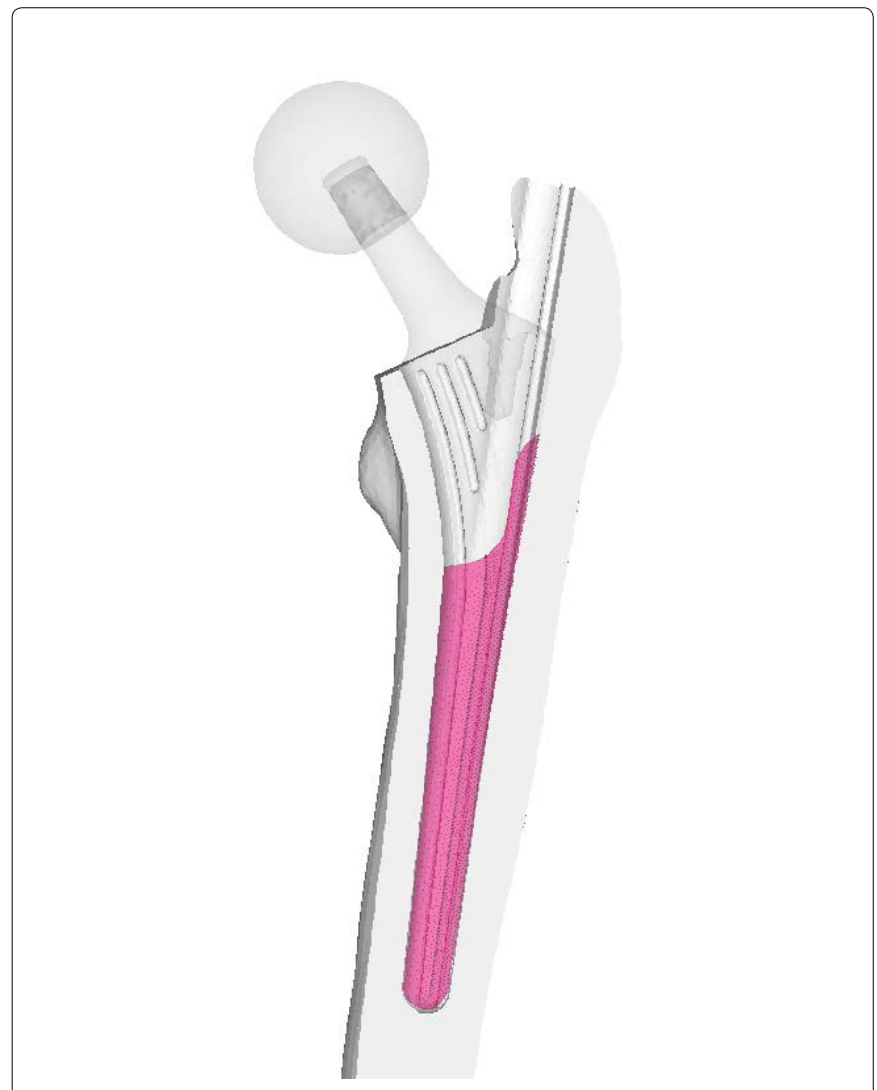

Figure 6: Contact option applied to implants (colored region).

al. was used in this experiment [30]. In the theoretical formulation, the rate of remodeling as the change of Young's modulus, $\mathrm{dE} / \mathrm{dt}$, was correlated with the strain energy density, $U$, as follows:

$$
\frac{d E}{d t}=\mathrm{C}\left(U-U_{n}\right)
$$

where $\mathrm{C}$ and $\mathrm{Un}$ are the remodeling constant and the site-specific homeostatic equilibrium strain energy density, respectively. In the present analysis, $C$ and Un were set at $1.2 \times 105$ and $0.011 \mathrm{MJ} / \mathrm{m}^{3}$, respectively.

\section{Results}

\section{Principal stress projections of intact model}

Preliminary principal stress projections predicted by FEA are shown in Figure 7. It was found that a distinct hypodense structure seen from the top of the femoral head tracing downwards to the calcar femorale of the femur on the CT image was represented as the minimum principal stress (red) on the FEA result. The epiphyseal line can also be seen in the CT scan, near the center of the femoral head. This is traceable by the maximum principal stress (blue). With the FEA result, it is also possible to view the five trabeculae groups: Principal tensile and compressive group (dashed), secondary tensile and compressive group (dotted), and the greater trochanter group. In the transverse plane shown in Figure $7 b$, it is possible to see both the epiphyseal line and the calcar femorale. With the coronal plane, principal stress projections can be drawn simply from the outer symmetry, as illustrated by Wolff. However, with 


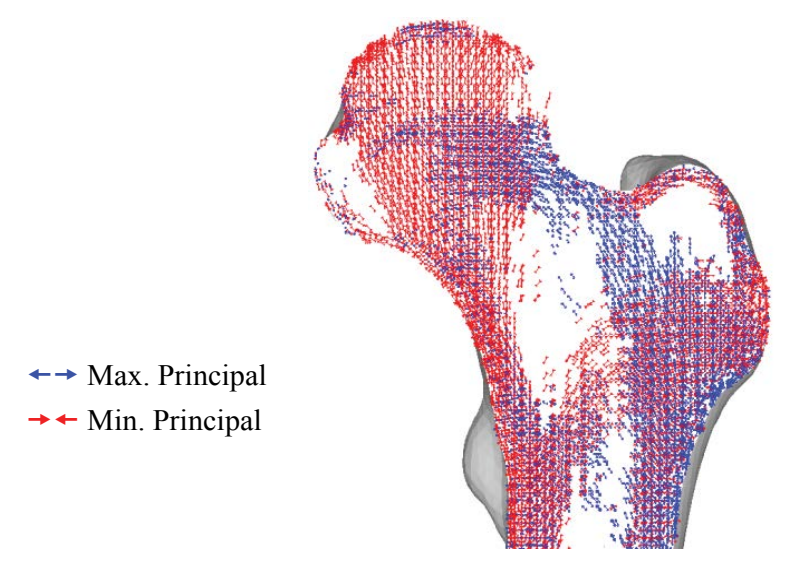

(a) Coronal projection

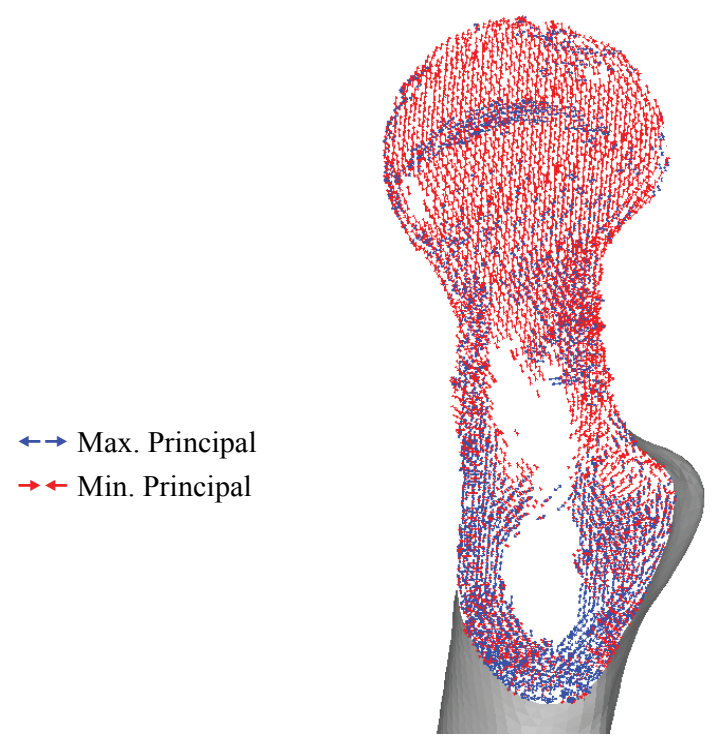

(b) Transverse projection

Figure 7: Principal stress projection in the femur.

the transverse plane, the stress projections are impossible to illustrate due to the loading being orthogonal to the plane. Despite of this, by utilizing the material properties, FEA has replicated the trabecular structure, as shown by the similarities in the transverse plane. These similarities indicate that 3D FEA can realistically predict trabeculae structure due to the stress projections being determined from material properties and not the symmetry. The intact bone was attested to be an appropriate control for the experiment.

\section{Principal stress projections of THA model}

The principal stress projections in the intact bone and THA models are shown in Figure 8. Obviously, none of the trabeculae groups remain in the THA model due to the absence of the femoral head. While the intact bone model indicates pure compressive loading along the medial region, the calcar femorale region shows tensile loading. Contrastingly, the lateral region shows increase in minimum principal stresses, indicating high compressive loading and densification of the trabecuale near the middle of the stem. The greater trochanter region does not significantly change regarding projection.

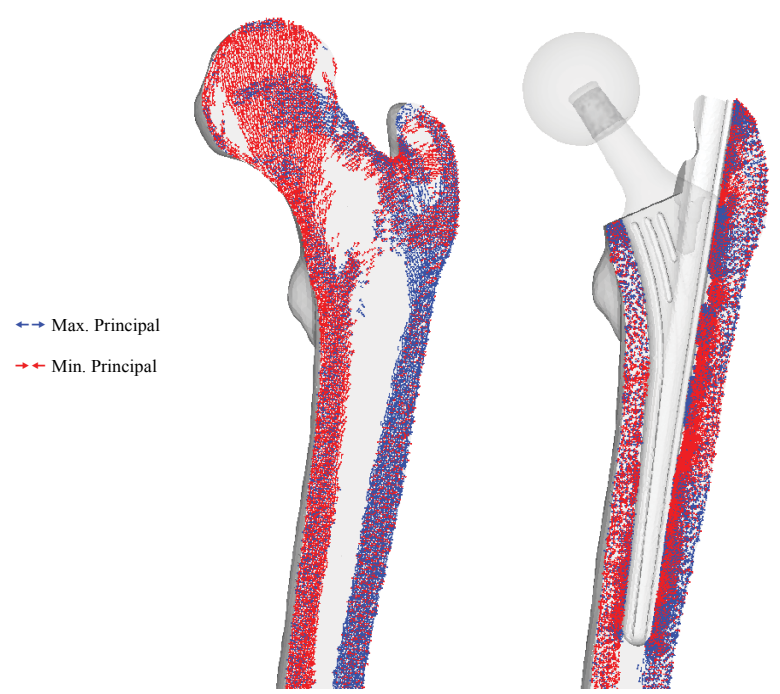

Figure 8: Preliminary principal stress projection (intact bone and THA).

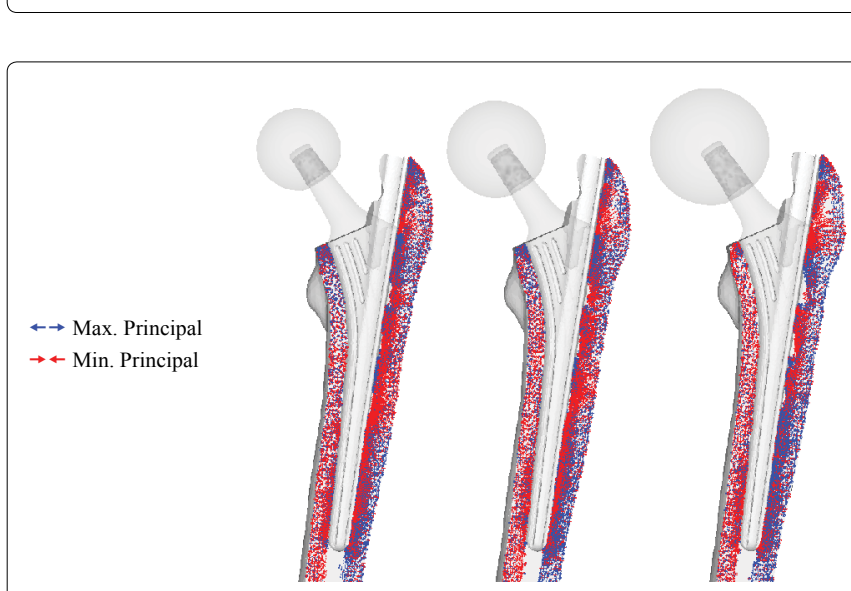

Figure 9: Preliminary principal stress projections with different head diameter $(\varnothing 34 \mathrm{~mm}, \varnothing 40 \mathrm{~mm}$, and $\varnothing 48 \mathrm{~mm})$.

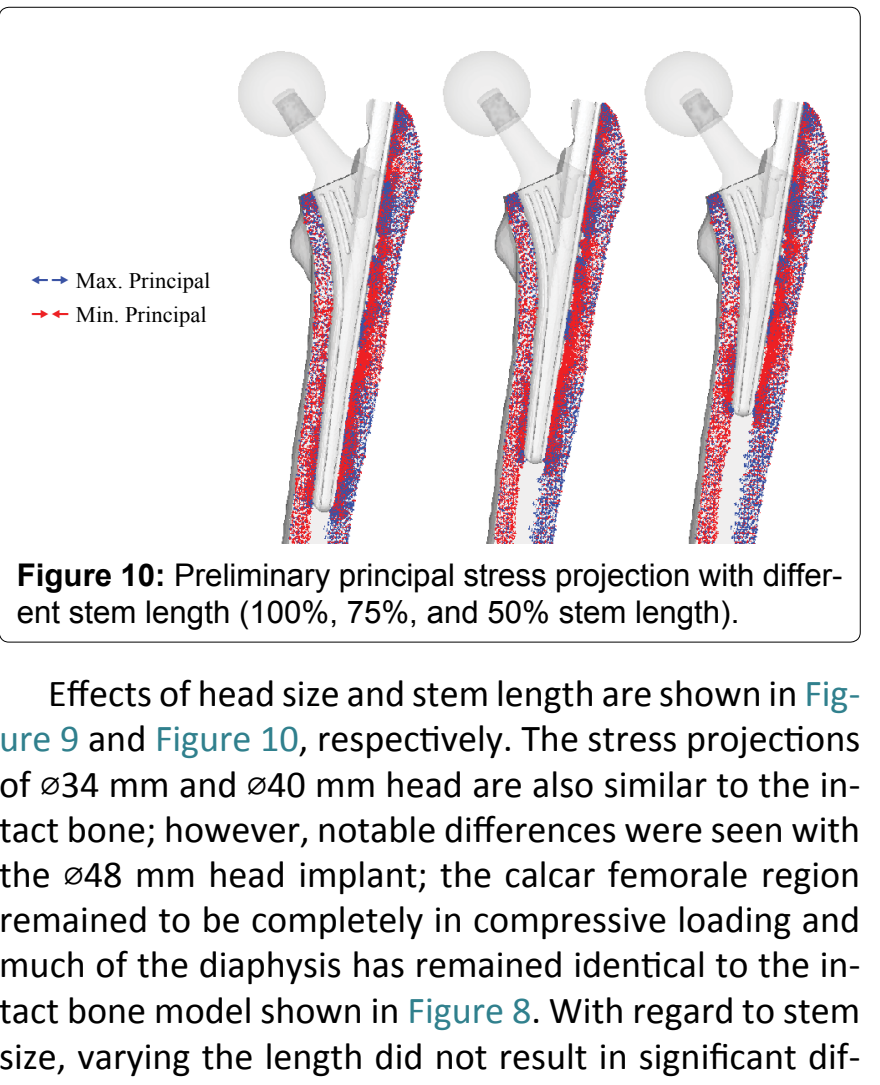


ferences as expressed in Figure 10. The loading region does appear to change in proportion to the stem length.

\section{Distribution of equivalent stress in THA and RHA models}

To assess the post-operative safety of the implant, the equivalent stress distribution of each model was investigated and compared to the intact bone model. Drucker-Prager yield criterion was chosen as bone is a brittle material and the compressive strength is higher than the tensile strength $[41,42]$. Equivalent stress distribution of the THA model reveals that the proximal lateral section experiences high stress, while the distal lateral section experiences low stress as shown in Figure 11. However, the stress increases around the implant tip. Proximal medial region, around the porous coating of the implant, also experiences high stress, although it appears to decrease distally. With increasing head size, the high stress in the diaphysis region seen in the standard THA model becomes absent as shown in Figure 12. The stresses in the greater trochanter also appear to

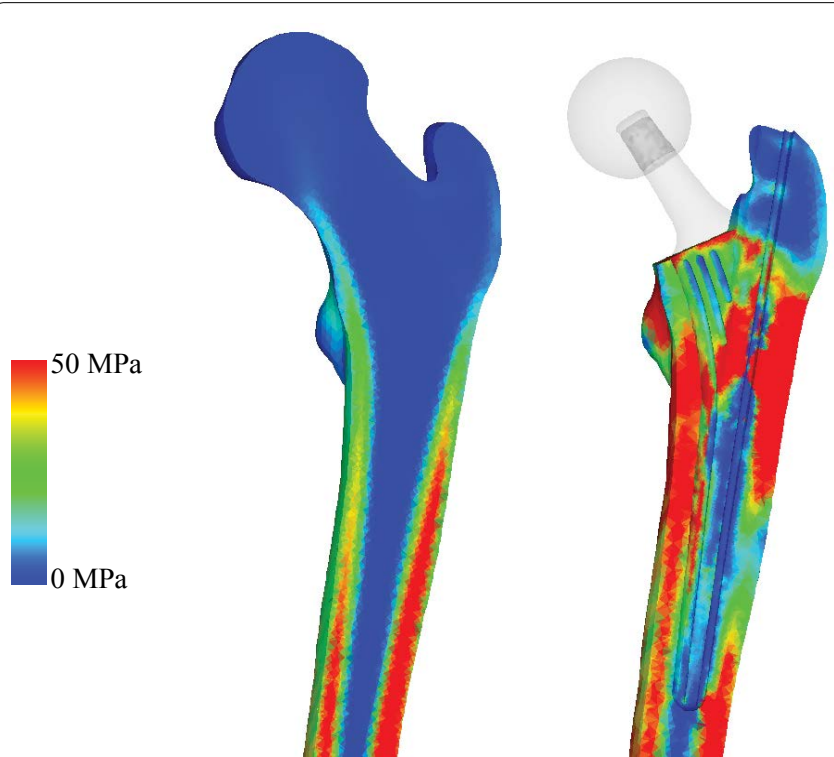

Figure 11: Drucker-Prager equivalent stress (intact bone and THA).

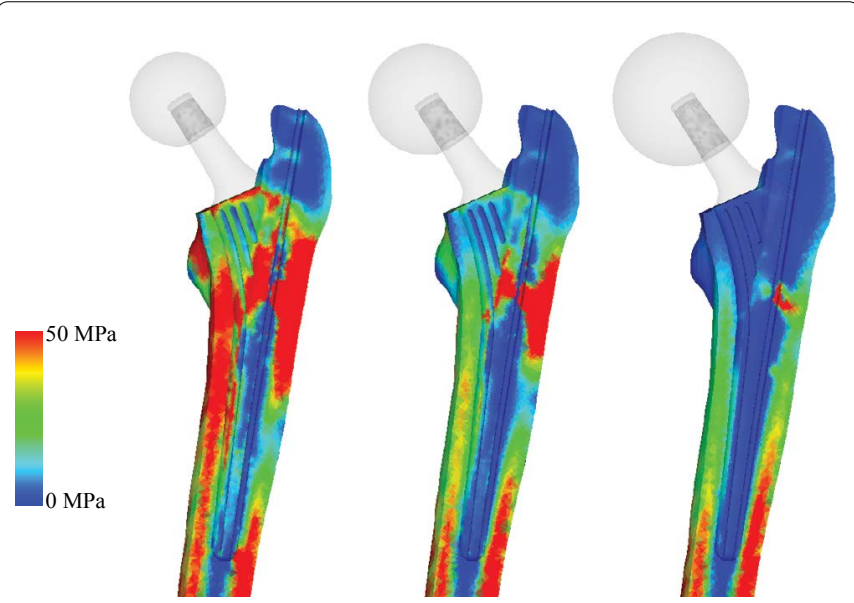

Figure 12: Drucker-Prager equivalent stress (ø34 mm, ø40 $\mathrm{mm}$, and $\varnothing 48 \mathrm{~mm}$ ). fade. With $\varnothing 48 \mathrm{~mm}$ head, the stress distribution closely resembles that of the intact bone. Furthermore, stress around the implant stem is not evident. Similar to the previous stress projection result, equivalent stress distribution did not differ to the THA stem despite differences in stem length as shown in Figure 13.

\section{Bone remodeling behavior}

Preliminary inspection is important to inspect the changes in the physiology of post-operative femur, but so far, only the mechanical aspects have been considered. In order to assess post-operative conditions and long-term outcomes, bone remodeling simulation is required. Bone remodeling was conducted on the standard implant with two main implants, THA implant with $\varnothing 48 \mathrm{~mm}$ head size, and THA implant with $50 \%$ stem length. The changes of principal stress projections at different times are shown in Figure 14 for the standard model, Figure 15 for the model with $\varnothing 48 \mathrm{~mm}$ head size, and Figure 16 for the model with $50 \%$ stem length. Thus, all implant models were remodeled until steady state was reached. Significant changes occur in the femoral head, as the disappearance of trabeculae groups causes formations of an unnatural trabeculae group as shown in Figure 17. The significant change with the THA implant model is the restoration of the medial and lateral projections, as well as the projections at the calcar femorale, which has changed back to compressive projections as shown in Figure 14. Collections of projections still remain along the surface of the implant and stem tip and continues until the remodeling reaches steady state. A clear area without projections also appears beneath the greater trochanter. In Figure 15 and Figure 16 , the key difference is the absence of stress projections around the implant tip.

\section{Bone mineral density}

Bone mineral density (BMD) was analyzed to link projections to trabeculae structure and the results are shown in Figure 17. For implant models, this was calculated at $\mathrm{t} 5$ corresponding to the steady state stage. $B M D$ of the intact bone was directly derived from the

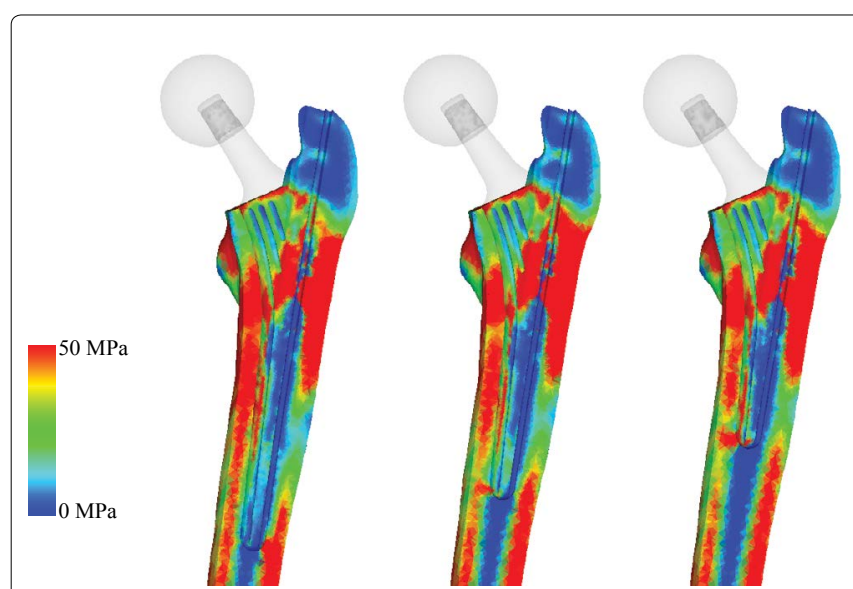

Figure 13: Drucker-Prager equivalent stress (100\%, 75\%, and $50 \%$ stem length). 

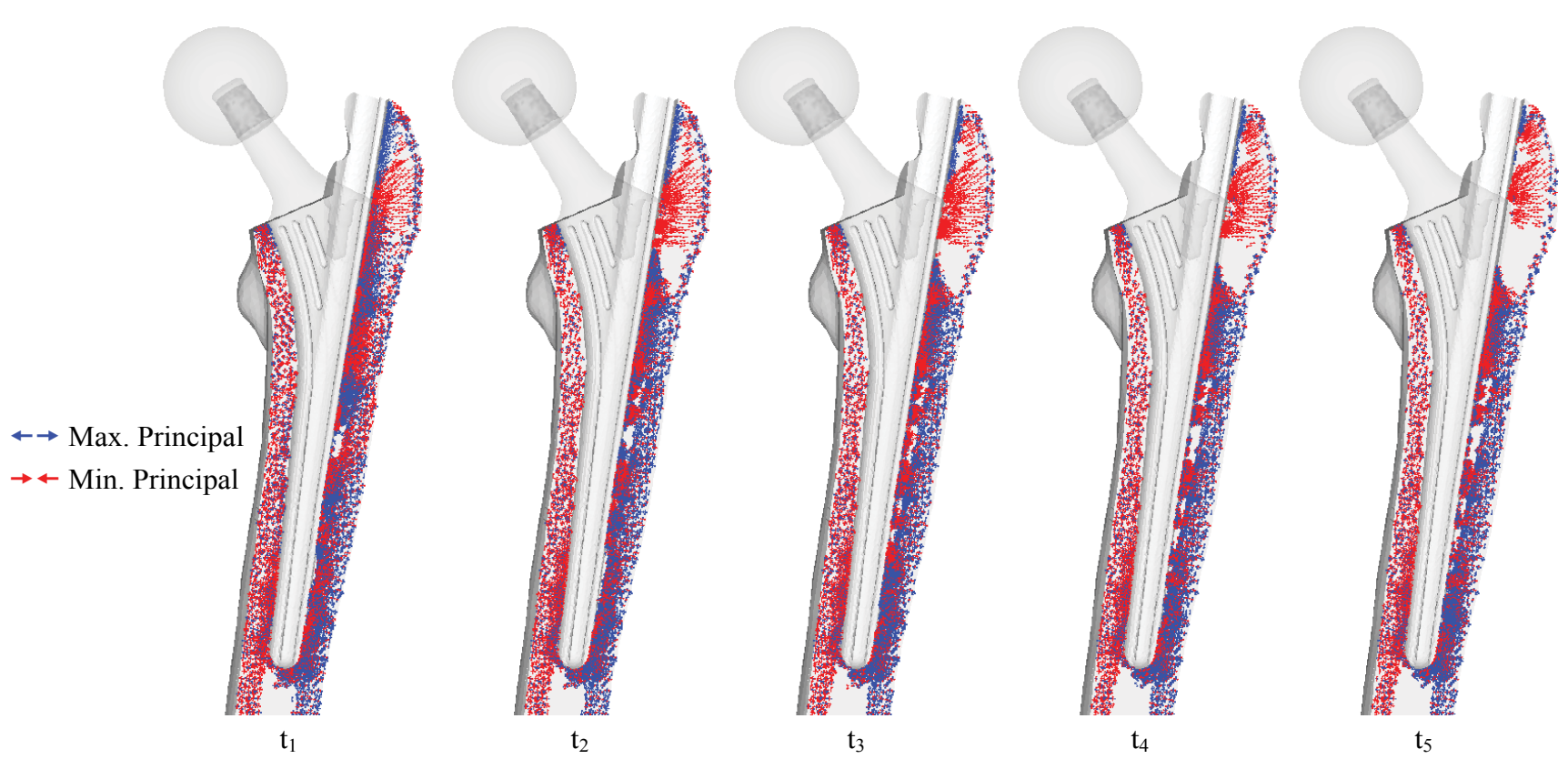

Figure 14: Bone remodeling with principal stress projections in the standard THA model.

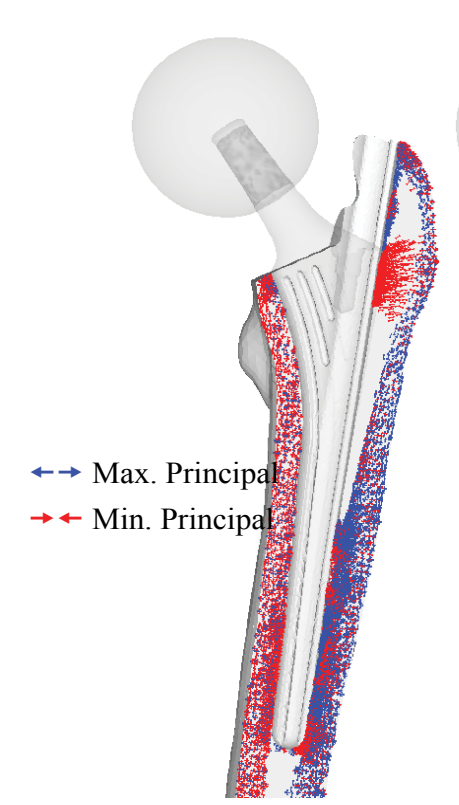

$t_{1}$

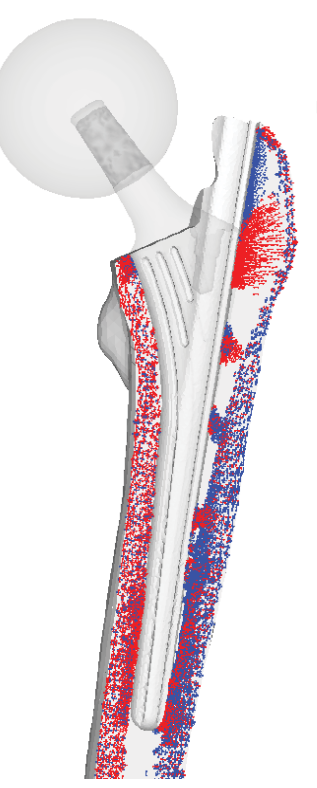

$\mathrm{t}_{2}$

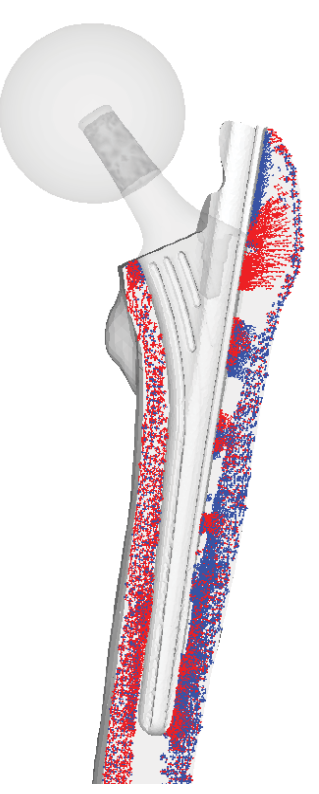

$t_{3}$

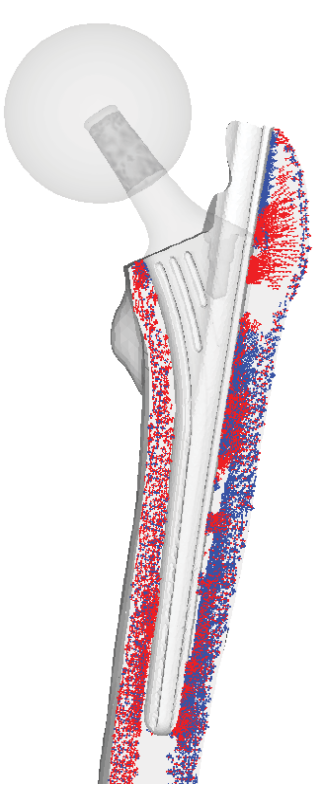

$\mathrm{t}_{4}$

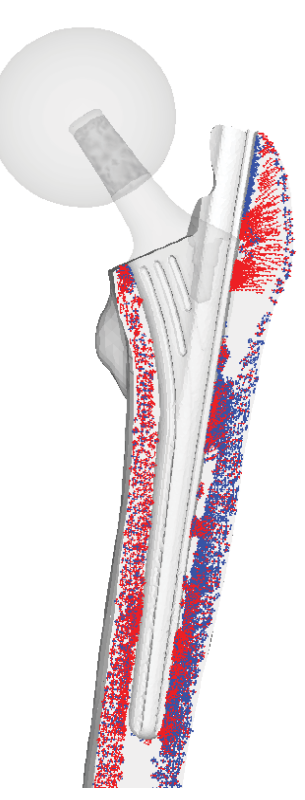

$t_{5}$

Figure 15: Bone remodeling with principal stress projections in the THA model with $\varnothing 48 \mathrm{~mm}$ head.

CT scans. From the BMD, the quality of the bone stock can be determined, which reflects on the strength of the bone as well as areas which may become the focal point for risks. Areas of high BMD correlates to high stress and/or stress shielding, and areas of low BMD reveals weakening of the bone and potential risk triggers. Like the stress projections, the BMD distribution is very similar to the intact bone. The diaphysis has maintained BMD on both the medial and lateral regions. However, there is a significant decrease in density in the femoral head. The distributions show that BMD is low around the porous coating, and high around the uncoated region. The stem tip also shows increased BMD. The shorter length model has a larger distribution in which density has increased. The stem tip on the larger head- ed implant, on the other hand, does not show signs of increased BMD.

\section{Discussion}

THA implants showed significant changes in the trabeculae structure in the preliminary experiment, apart from the $\varnothing 48 \mathrm{~mm}$ head implant, which was able to maintain the characteristics of the diaphysis. When the Drrucker-Prager equivalent stress was inspected, THA models revealed extremely high stress areas around the proximal region. Again, the distributions of the larger head-sized implant were akin to the intact bone model.

While bone remodeling simulation has revealed that the diaphysis returns to its original state, the lat- 


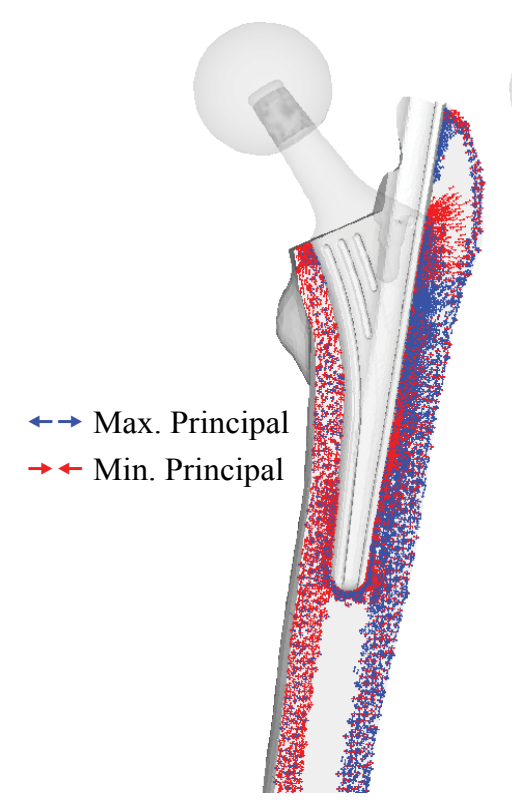

$t_{1}$

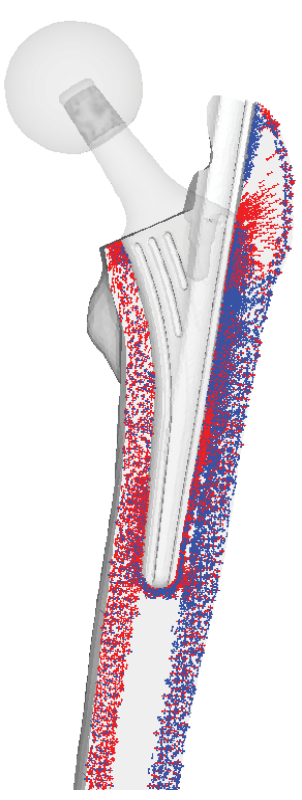

$\mathrm{t}_{2}$

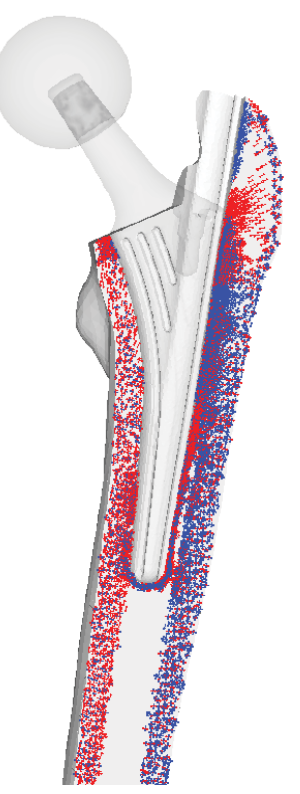

$t_{3}$

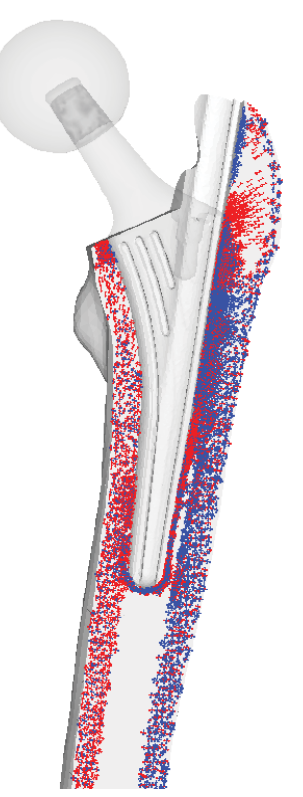

$t_{4}$

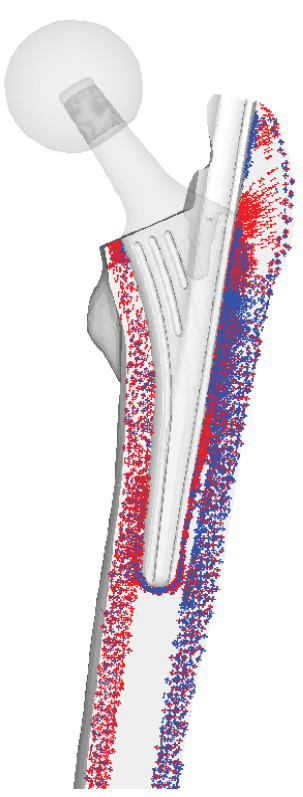

$t_{5}$

Figure 16: Bone remodeling with principal stress projections in the THA model with $50 \%$ stem length.
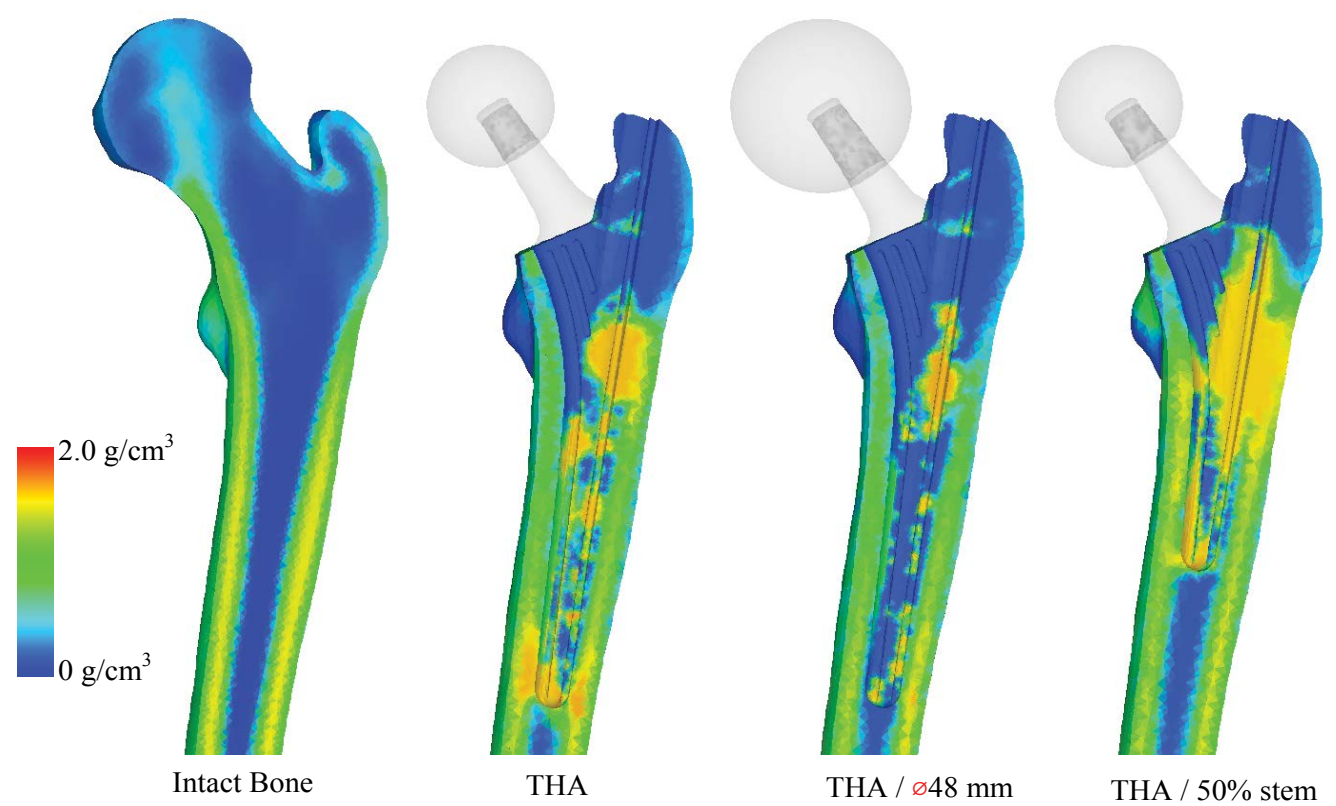

Figure 17: Bone mineral density after bone remodeling.

eral proximal region, below the greater trochanter, and the tip of the implant stem, remains to be an issue. This finding was also reflected with the BMD analysis, where increased bone density was found in identical locations. Such characteristics where two highly densified areas separated by low density region could lead to peri-prosthetic fracture. These findings were found for the standard THA model and the shortened THA model but were not consistent with the larger head implant model. In all cases, the implant closely matched the results of the intact bone model.

The design concept behind HRA is justifiable as a larger head size increases the ROM of the joint. However, long-term computational assessments and BMD results have shown that it does not fare well when compared
THA implants. THA implants, despite of differences from the intact bone, does maintain BMD throughout the diaphysis. Shorter stem length has also showed similar results with the standard THA. Furthermore, a larger head has shown to be superior to the standard THA. From a medical standpoint, decreasing the stem size correlates with conservation of bone mass, and increasing head size correlates with increased ROM.

\section{Conclusions}

A novel process to simulate long-term biomechanics of femur with THA implant has been proposed. FEA has provided to be an integral tool to realistically model the biomechanics of the femur, with the use of realistic physiological loading conditions. Computational mod- 
eling of bone adaptation has also predicted the longterm physiological outcome of a femur with implant and conducting risk assessments have revealed possible fracture areas due to long-term remodeling. Using this process, implant designers can investigate the femur physiology of design, and determine long-term effects by performing bone adaptation and risk assessment, before the design enters production. Medical doctors can also benefit from this process during pre-operative planning stage. An ideal implant can be chosen purely from the patient's femur's biomechanics, and longterm assessments can estimate when post-operative follow-up should be performed before serious damages occur. The research has closely achieved clinical results with engineering methodology. It has also shown that mechanical assessment is not enough to effectively evaluate the implant. Biomechanical assessment must be conducted to fully establish the safety of a design.

\section{References}

1. Gomez PF, Morcuende JA (2005) Early attempts at hip arthroplasty - 1700s TO 1950s. lowa Orthop J 25: 25-29.

2. Dowson D, Hardaker C, Flett M, Isaac GH (2004) A hip joint simulator study of the performance of metal-on-metal joints. J Arthroplasty 19: 124-130.

3. M Porter, K Tucker, R Beaumont, E Young, O Forsyth, et al. (2012) National Joint Registry for England and Wales, 9th Annual Report.

4. Malik A, Maheshwari A, Dorr LD (2007) Impingement with total hip replacement. J Bone Joint Surg Am 89: 1832-1842.

5. Kelley SS, Lachiewicz PF, Hickman JM, Paterno SM (1998) Relationship of femoral head and acetabular size to the prevalence of dislocation. Clin Orthop Relat Res 355: 163-170.

6. Lavigne M, Ganapathi M, Mottard S, Girard J, Vendittoli PA (2011) Range of motion of large head total hip arthroplasty is greater than $28 \mathrm{~mm}$ total hip arthroplasty or hip resurfacing. Clin Biomech (Bristol, Avon) 26: 267-273.

7. Joshi MG, Advani SG, Miller F, Santare MH (2000) Analysis of a femoral hip prosthesis designed to reduce stress shielding. J Biomech 33: 1655-1662.

8. Abdul H Abdullah, Mohd N Mohd Asri, Mohd S Alias, Tardan Giha (2010) Finite element analysis of cemented hip arthroplasty: Influence of stem tapers. In. Proceedings of the International MultiConference of Engineers and Computer Scientists vol III.

9. Behrens BA, Wirth CJ, Windhagen H, Nolte I, Meyer-Lindenberg A, et al. (2008) Numerical investigations of stress shielding in total hip prostheses. Proceedings of the Institution of Mechanical Engineers, Part $\mathrm{H}$ : Journal of Engineering in Medicine 222: 593-600.

10. Abdul Halim Abdullah, Mohd Asri Mohd Nor, Alias Mohd Saman (2009) Stress and Strain Distribution in Cemented Total Hip Arthroplasty for Walking Load Case. 2009 International Conference on Computer Technology and Development 260-263.

11. MIZ Ridzwan, S Shuib, AY Hassan, AA Shokri, MN Mohamad Ibrahim (2007) Problem of Stress Shielding and Improvement to the Hip Implant Design - A Review. Journal of Medical Sciences 7: 460-467.

12. Kowalczyk P (2010) Simulation of orthotropic microstructure remodelling of cancellous bone. J Biomech 43: 563-569.

13. JM Garcia, M Doblare, J Cegonino (2002) Bone remodelling simulation: A tool for implant design. Computational Materials Science 25: 100-114.

14. Herrera A, Panisello JJ, Ibarz E, Cegoñino J, Puértolas JA, et al. (2007) Long-term study of bone remodelling after femoral stem: A comparison between dexa and finite element simulation. J Biomech 40: 3615-3625.

15. Scannell PT, Prendergast PJ (2009) Cortical and interfacial bone changes around a non-cemented hip implant: simulations using a combined strain/damage remodelling algorithm. Med Eng Phys 31: 477-488.

16. J Belinha, RM Natal Jorge, LMJS Dinis (2012) Bone tissue remodelling analysis considering a radial point interpolator meshless method. Engineering Analysis with Boundary Elements 36: 1660-1670.

17. Kerner J, Huiskes $\mathrm{R}$, van Lenthe $\mathrm{GH}$, Weinans $\mathrm{H}$, van Rietbergen $B$, et al. (1999) Correlation between pre-operative periprosthetic bone density and post-operative bone loss in THA can be explained by strain-adaptive remodelling. $\mathrm{J}$ Biomech 32: 695-703.

18. Waide V, Cristofolini L, Stolk J, Verdonschot N, Toni A (2003) Experimental investigation of bone remodelling using composite femurs. Clin Biomech 18: 523-536.

19. Skedros JG, Brand RA (2011) Biographical sketch: Georg Hermann von Meyer (1815-1892). Clin Orthop Relat Res 469: 3072-3076.

20. Skedros JG, Baucom SL (2007) Mathematical analysis of trabecular 'trajectories' in apparent trajectorial structures: the unfortunate historical emphasis on the human proximal femur. J Theor Biol 244: 15-45.

21. Keyak JH, Rossi SA, Jones KA, Skinner HB (1998) Prediction of femoral fracture load using automated finite element modeling. J Biomech 31: 125-133.

22. Bergmann G, Graichen F, Rohlmann A, Bender A, Heinlein $B$, et al. (2010) Realistic loads for testing hip implants. Biomed Mater Eng 20: 65-75.

23. Bergmann G, Deuretzbacher G, Heller M, Graichen F, Rohlmann A, et al. (2001) Hip contact forces and gait patterns from routine activities. J Biomech 34: 859-871.

24. Simões JA, Vaz MA, Blatcher S, Taylor M (2000) Influence of head constraint and muscle forces on the strain distribution within the intact femur. Med Eng Phys 24: 243.

25. Bitsakos C, Kerner J, Fisher I, Amis AA (2005) The effect of muscle loading on the simulation of bone remodelling in the proximal femur. J Biomech 38: 133-139.

26. Fyhrie DP, Schaffler MB (1995) The adaptation of bone apparent density to applied load. J Biomech 28: 135-146.

27. Weinans H, Huiskes R, Grootenboer HJ (1992) Effects of material properties of femoral hip components on bone remodeling. J Orthop Res 10: 845-853.

28. Turner CH, Anne V, Pidaparti RM (1997) A uniform strain criterion for trabecular bone adaptation: Do continuum-level strain gradients drive adaptation? J Biomech 30: 555-563.

29. M. Mullender, B Van Rietbergen, $P$ Rüegsegger, $R$ Huiskes (1998) Effect of mechanical set point of bone cells on mechanical control of trabecular bone architecture. Bone 22: 125-131.

30. Huiskes R, Weinans H, Grootenboer HJ, Dalstra M, Fudala B (1987) Adaptive bone-remodeling theory applied to prosthetic-design analysis. J Biomech 20: 1135-1150. 
31. San Antonio T, Ciaccia M, Müller-Karger C, Casanova E (2012) Orientation of orthotropic material properties in a femur FE model: A method based on the principal stresses directions. Med Eng Phys 34: 914-919.

32. C Dick, J Georgii, R Burgkart, R Westermann (2009) Stress tensor field visualization for implant planning in orthopedics. IEEE transactions on visualization and computer graphics 15: 1399-1406.

33. Smith AJ, Dieppe P, Vernon K, Porter M, Blom AW (2012) Failure rates of stemmed metal-on-metal hip replacements: Analysis of data from the National Joint Registry of England and Wales. Lancet 379: 1199-1204.

34. Smith AJ, Dieppe P, Howard PW, Blom AW (2012) Failure rates of metal-on-metal hip resurfacings: Analysis of data from the National Joint Registry for England and Wales. Lancet 380: 1759-1766.

35. Chang PB, Robie BH, Bartel DL (1999) Preclinical cost analysis of orthopaedic implants: A custom versus standard cementless femoral component for revision total hip arthroplasty. J Biomech 32: 1309-1318.

36. M Baleani, M Viceconti, R Muccini, M Ansaloni (2000) Endurance verification of custom-made hip prostheses. International Journal of Fatigue 22: 865-871.
37. Bert JM (1996) Custom total hip arthroplasty. J Arthroplasty 11: $905-915$

38. Götze C, Steens W, Vieth V, Poremba C, Claes L, et al. (2002) Primary stability in cementless femoral stems: custom-made versus conventional femoral prosthesis. Clin Biomech (Bristol, Avon) 17: 267-273.

39. Kawate K, Ohneda Y, Ohmura T, Yajima H, Sugimoto K, et al. (2009) Computed tomography-based custom-made stem for dysplastic hips in Japanese patients. J Arthroplasty $24: 65-70$.

40. Heller MO, Bergmann G, Kassi JP, Claes L, Haas NP, et al. (2005) Determination of muscle loading at the hip joint for use in pre-clinical testing. J Biomech 38: 1155-1163.

41. Bessho M, Ohnishi I, Matsuyama J, Matsumoto T, Imai K, et al. (2007) Prediction of strength and strain of the proximal femur by a CT-based finite element method. J Biomech 40: 1745-1753.

42. Wakao N, Harada A, Matsui $Y$, Takemura M, Shimokata $H$, et al. (2009) The effect of impact direction on the fracture load of osteoporotic proximal femurs. Med Eng Phys 31: 1134-1139. 